\title{
Genetix
}

Take the lead

\section{Rapid automated selection of mammalian cell colonies by cell surface protein expression}

\author{
Genetix's unique ClonePix FL technology uses our ability to grow mammalian cells into clonal colonies \\ suspended in semisolid media, and image these colonies using fluorescence assays and markers. Here \\ we describe a new application for this technology in the isolation of clonal cell populations based on \\ their expression of cell surface proteins.
}

The ability to select cells based on surface protein expression is of great use in establishing cell lines expressing receptor(s) of interest for downstream phenotyping or genotyping, for use in basic discovery research, or as cell lines for cell-based assays and screening. Historically such separation of cells has been achieved using fluorescence-activated flow cytometry to sort cells based on protein expression. Using our fluorescence imaging technology, we have developed a high-powered alternative to flow cytometry that not only allows users to select the highest expressers for a particular receptor, or combination of receptors or other cell surface proteins, but also yields monoclonal populations of cells.

For this application of the ClonePix FL technology, single cells are plated at a density of 300-1,000 cells/ml (depending on the cell line) in Genetix CloneMatrix-based semisolid medium (using the appropriate liquid medium base for each cell line under investigation) into black-walled Genetix PetriWell-6 plates and are allowed to grow into colonies of $50-100$ cells per colony at $37^{\circ} \mathrm{C}(7-14 \mathrm{~d}$, depending on the cell line). When the colonies reach this optimal size, a fluorescent antibody to the receptor of interest is sprayed onto the semisolid medium using Genetix Atomiser bottles to achieve an even covering of antibody solution over the well. Subsequent incubation for 24 $48 \mathrm{~h}$ allows the antibody to diffuse through the medium and bind to the colonies expressing the receptors of interest. Colonies of cells are then imaged by ClonePix FL using fluorescence and analyzed using ClonePix FL's dedicated software. The selected fluorescent colonies are automatically picked from the semisolid medium and dispensed into the wells of a 96-well destination plate. In the following proof-ofprinciple studies, the picked clonal populations of cells were analyzed after picking to confirm good growth and viability after the picking process.

\section{Edmund N C Newman \& Dominick Whitney}

Genetix Ltd., Queensway, New Milton, Hants, BH25 5NN, UK. Correspondence should be addressed to E.N.C.N. (edmund.newman@genetix.com).
Selection of endogenously expressed surface proteins We have optimized our semisolid media to allow growth of a variety of cells into suspended clonal colonies of 50-100 cells in less than $14 \mathrm{~d}$. This allows us to select cells from a mixed or heterogeneous population for their expression of endogenous cell surface proteins, using fluorescently conjugated antibody to the protein of interest (Fig. 1).

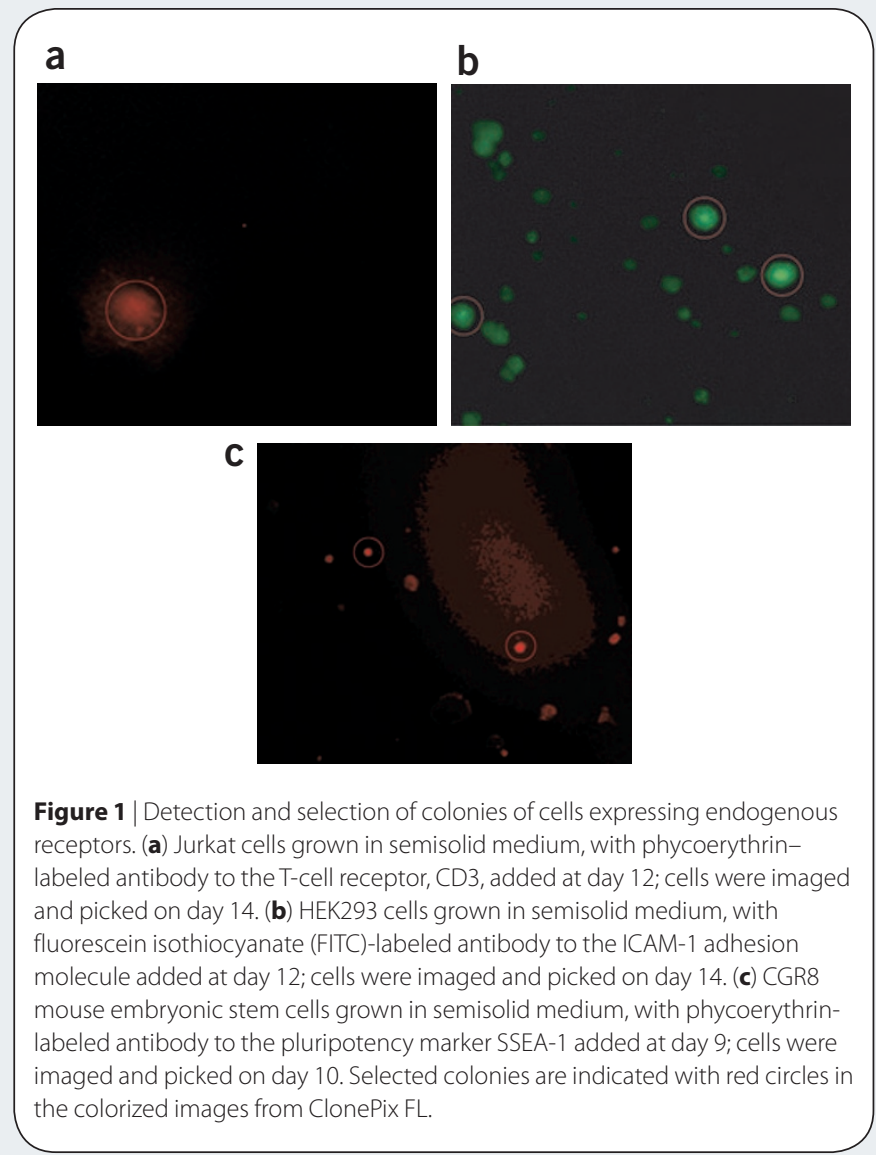


APPLICATION NOTES

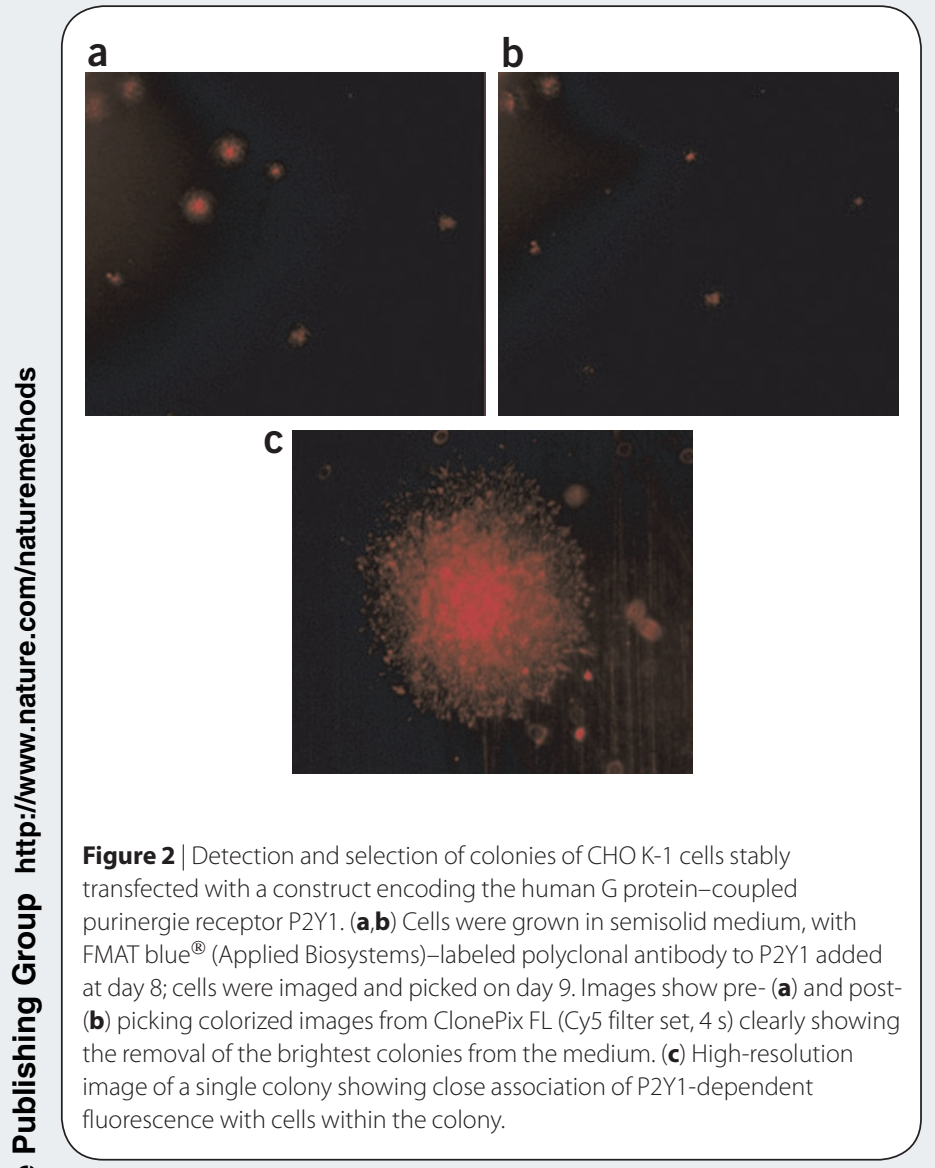

Selection of transfected, exogenous cell-surface proteins

We have also extended this technology to the selection of cell lines transfected with a construct encoding a receptor of interest (Fig. 2). With the aid of an antibody to this receptor, the ClonePix FL technology allows the highest expressing colonies from a population of transient or stable transfectants to be selected based on fluorescence intensity (and therefore receptor expression levels). This is of great importance in generating reporter cell lines for screening.

\section{Selection using epitope-tagged receptors}

During target-discovery research for new receptors and drug targets, it may not be possible to generate or acquire an antibody to the new receptor of interest. As an alternative, we validated our technology for picking colonies of transfected cells expressing receptors with extracellular epitope tags. In this case, we quantified expression using fluo-

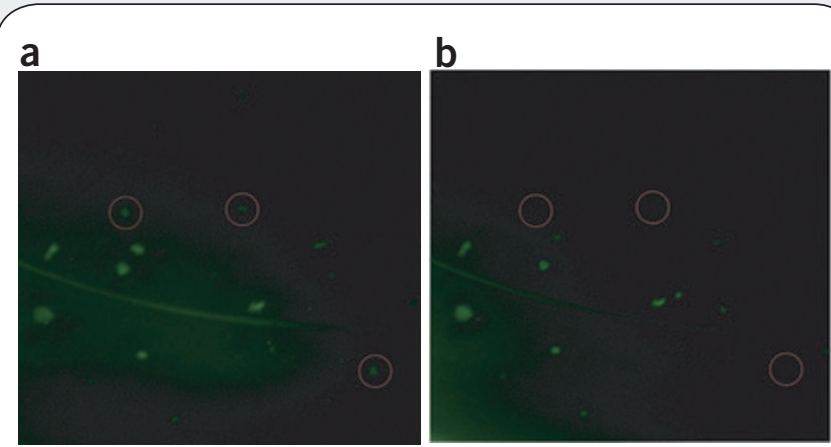

Figure 3 | Detection and selection of colonies of CHO K-1 cells that have been stably transfected with a Flag-tagged human drug target receptor. $(\mathbf{a}, \mathbf{b})$ Cells were grown in semisolid media, with FITC-labeled anti-Flag monoclonal added at day 8; the cells were imaged and picked on day 10. Images show pre- (a) and post- (b) picking colorized images from ClonePix FL (FITC filter set, $5 \mathrm{~s}$ ) showing the removal of the brightest colonies from the medium.

rescently conjugated antibody to an epitope tag. An example of such an experiment, using a Flag-tagged transfected receptor, is shown in Figure 3.

\section{Conclusion}

By combining the fluorescent detection of protein expression with the 'cloning' of cells (to give monoclonal populations) into one single step, ClonePix FL technology can greatly improve the time requirement, labor costs and overall efficiency of selecting clonal cell lines based on surface protein expression. Our new method of growing colonies of cells before automated analysis and picking results in much higher viability of 'sorted' cells, allowing us to identify one colony of interest from as many as 3,000 clones. This application of our established technology will greatly improve the quality and efficiency of cell-line development.

\section{Antibody reagents}

To support this application, Genetix has developed an extensive panel of fluorescently labeled antibodies to a range of human cell-surface proteins, all of which come with our unique atomizer bottles for easy and simple application to our semisolid media. For more information please contact info@genetix.com.

\footnotetext{
This article was submitted to Nature Methods by a commercial organization and has not been peer reviewed. Nature Methods takes no responsibility for the accuracy or otherwise of the information provided.
} 